\title{
Data Acquisition in a High Harmonic Generation Lab and at LCLS
}

\author{
Takako Hirokawa \\ Office of Science, Science Undergraduate Laboratory Internship (SULI) \\ University of Colorado-Boulder \\ SLAC National Accelerator Laboratory \\ Menlo Park, CA
}

August 20, 2010

Prepared in partial fulfillment of the requirements of the Office of Science, Department of Energy's Science Undergraduate Laboratory Internship under the direction of Markus Gühr \& Brian K. McFarland at the Stanford PULSE Institute, SLAC National Accelerator Laboratory.

Participant:

Signature

Research Advisor:

\author{
Signature
}




\section{TABLE OF CONTENTS}

$\begin{array}{ll}\text { Abstract } & \text { ii }\end{array}$

$\begin{array}{ll}\text { Introduction } & 1\end{array}$

$\begin{array}{ll}\text { Materials and Methods } & 3\end{array}$

$\begin{array}{ll}\text { Results } & 7\end{array}$

$\begin{array}{lr}\text { Discussion and Conclusions } & 8\end{array}$

$\begin{array}{lr}\text { Acknowledgments } & 9\end{array}$

$\begin{array}{lr}\text { References } & 9\end{array}$ 


\begin{abstract}
Data Acquisition in a High Harmonic Generation Lab and at LCLS. TAKAKO HIROKAWA (University of Colorado-Boulder, Boulder, CO 80310) MARKUS GÜHR \& BRIAN K. MCFARLAND (Stanford PULSE Institute, SLAC National Accelerator Laboratory, Stanford, CA 94025)
\end{abstract}

In this paper, we examine data acquisition in a high harmonic generation (HHG) lab and preliminary data analysis with the Cyclohexadiene Collaboration at the Linac Coherent Lightsource (LCLS) at SLAC National Accelerator Laboratory. HHG experiments have a large number of parameters that need to be monitored constantly. In particular, the pressure of the target is critical to HHG yield. However, this pressure can fluctuate wildly and without a tool to monitor it, it is difficult to analyze the correlation between HHG yield and the pressure. I used the Arduino microcontroller board and created a complementary MATLAB graphical user interface (GUI), thereby enhancing the ease with which users can acquire time-stamped parameter data. Using the Arduino, it is much easier to match the pressure to the corresponding HHG yield. Collecting data by using the Arduino and the GUI is flexible, user-friendly, and cost-effective. In the future, we hope to be able to control and monitor parts of the lab with the Arduino alone. While more parameter information is needed in the HHG lab, we needed to reduce the amount of data during the cyclohexadiene collaboration. This was achieved by sorting the data into bins and filtering out unnecessary details. This method was highly effective in that it minimized the amount of data without losing any valuable information. This effective preliminary data analysis technique will continue to be used to decrease the size of the collected data. 


\section{INTRODUCTION}

Data acquisition is an integral part of any experiment. Without a tool to collect data, the experiment cannot produce results. This tool should be straight forward so that one can maximize use of one's time on tasks such as the data analysis. Each experiment has particular requirements for the data acquisition system. Though experiments in a high harmonic generation (HHG) lab and experiments using the Linac Coherent Lightsource (LCLS) X-ray free electron laser (FEL) at SLAC National Accelerator Laboratory revolve around the same general principle — using light pulses of extremely short wavelength and duration — the specifications of the data acquisition systems can differ vastly.

\section{Motivation}

Laser pulses of femtosecond $\left(10^{-15}\right.$ seconds $)$ to attosecond $\left(10^{-18}\right.$ seconds $)$ duration and extremely short wavelength are used to study molecular electronic structure and dynamics. One way to produce these short laser pulses is to use a free electron laser (FEL) for X-rays that generates wavelengths at the atomic scale, as demonstrated with the LCLS. However, an FEL requires an enormous amount of equipment, room, and therefore funding to generate these X-ray beams. These requirements have reduced the amount of available user time. Alternatively, the so-called high harmonic generation (HHG) can be used to create short soft

X-ray laser pulses that are much weaker in energy than FEL pulses. This second technology is widely used and is available in many laser labs in a table-top setting.

\section{$H H G$ Background}

HHG is achieved by ionizing atoms and letting their electrons recombine within several hundred attoseconds. This is accomplished by sending very short, strong infrared (IR) light pulses into a gas jet. Semi-classically speaking, $[1,2,3]$ if the pulse's electric field is of the 
same magnitude as that between an electron and the nucleus, the electric field bends the Coulomb potential such that one side of the potential well slopes up slightly while the other side slopes down (Fig. 1a). As a result, the electron tunnels out of the potential well and accelerate away from the ion. Since the laser field is oscillating very quickly, the potential flips rapidly so that the electron accelerates back into the center of the well, thereby gaining kinetic energy (Fig. 1b). When the it recombines with the atom, the electron emits its excess kinetic energy in the form of a photon (Fig. 1c). This recombination process takes a few hundred attoseconds, so the photons are released in an attosecond light pulse. The harmonic generation process repeats coherently on every half cycle of the strong driving laser field, and a train of attosecond pulses emerges. In the spectral domain, this series of pulses will appear as a discretization in the form of odd harmonics.

An experimental setup for a typical HHG experiment consists of many components. Figure 2 shows the sketch of a laboratory setup [4]. The harmonics are produced during the IR laser's interaction with the gas jet. They propagate through vacuum because they would be absorbed by air. The harmonic spectrum is dispersed by a grating spectrometer and detected by a soft x-ray CCD camera. These parts of the acquisition system have existed in the laboratory prior to this summer. I set up a new acquisition system that continuously logs crucial parameters of the experiment such as laser intensity and gas jet pressure. Since the gas jet pressure can fluctuate wildly and correlates with HHG yield, being able to monitor this parameter is extremely critical. The data acquisition system hard- and software had to be extremely flexible and needed to interface with MATLAB.

\section{Cyclohexadiene Collaboration at LCLS}

During my stay at SLAC, I was part of the data acquisition subgroup in a user team at the LCLS. At this facility, femtosecond X-ray pulses are created by accelerating electrons 
to high kinetic energies before they are injected into the undulators. Undulators consist of alternating magnetic structures that bend the electrons' path (Fig. 3). As the electrons change direction, they emit radiation in the form of X-ray photons. After they travel past the undulators, the electrons are sent to an electron dump, while the X-ray beam continues to propagate to be used for experiments.

The experiment conducted by the cyclohexadiene collaboration group at LCLS this summer investigated the opening of the cyclohexadiene ring-molecule after ultraviolet (UV) radiation. This was done by exciting the molecule with an ultrashort UV pulse and then probing it with a time delayed pulse of the LCLS to ionize and Coulomb explode the molecule. An electric field is imposed on the interaction region so that after the x-ray hits the cyclohexadiene, the ions and electrons from the shattered molecule are directed onto detectors. One can monitor the velocity, as well as the momentum, of the ions through velocity map imaging (VMI). An ion time of flight (iTOF) spectrometer is used to identify the mass-to-

charge ratio of the fragments. The ions are accelerated onto an ion sensitive detector capable of detecting single ions (see VMI in Fig. 4). By documenting the iTOF and the VMI, one can reconstruct the transient configuration of the molecule on a femtosecond time scale. [5, 6]

\section{MATERIALS AND METHODS}

\section{Materials and Methods in the HHG Lab}

\section{The Arduino}

The Arduino reads in sensor data and relays these values to the computer to which it is connected. The computer logs and plots the data. The Arduino has six analog inputs, 14 digital input and output pins, and a USB connection (Fig. 5). The analog inputs take DC voltage signals between 0 and $5 \mathrm{~V}$ and digitize them with 10 bit resolution. The Arduino is 
currently housed in a box that includes a small breadboard for signal preconditioning. Figure 6 shows the Arduino and the breadboard in the box. Sensors that measure the parameters of the lab such as the pressure in the vacuum chamber or the laser power can be connected to the Arduino directly via shielded BNC connectors. Oftentimes, the sensors output a voltage range that exceeds the $0-5 \mathrm{~V}$ range that the Arduino can accept. As a result, the signal has to be conditioned. In the case of the pressure gauge, a voltage divider is used to condition the signal such that its $0-10 \mathrm{~V}$ range is limited to a $0-5 \mathrm{~V}$ range. The Arduino has a clock speed of $16 \mathrm{MHz}$. The chip on the Arduino has flash memory of up to $16 \mathrm{~KB}$ onto which one can store a program as firmware [7]. This program is executed by the Atmel microcontroller chip on the Arduino board. [8]

The Arduino developers have created a programming environment that facilitates programming the microcontroller. Instead of using an assembly language, a convenient simple Java dialect is implemented [9]. For this project, I wrote a sketch onto the Arduino that allows it to recognize a signal from the computer that is transmitted via the serial port. The Arduino responds by sending a conditioned sensor value back to the computer via the same serial port. The following is an example of the sketch stored on the Arduino:

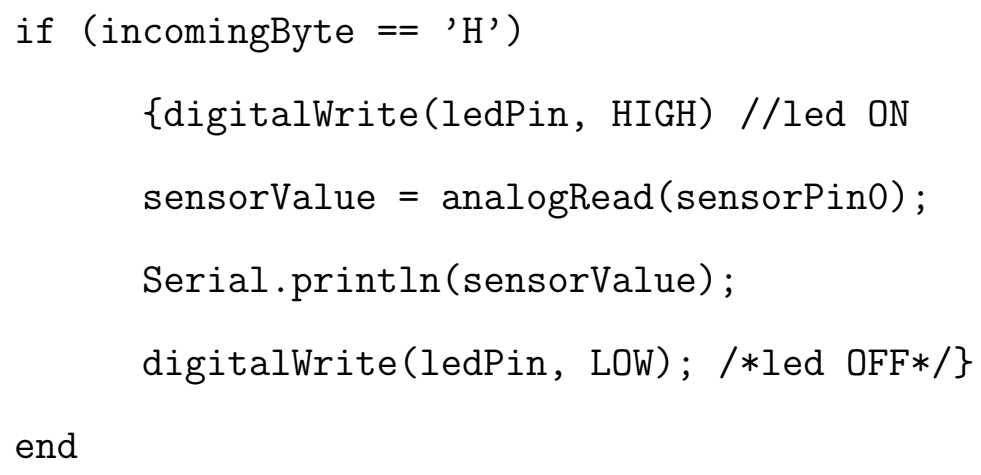

In this code example, incomingByte is the letter that the computer sends to the Arduino. In the second line of the loop, the Arduino reads analog input 0 and in the third 
line, it sends that value to the computer. The first and fourth lines turn an LED on the Arduino board on and off, respectively.

\section{MATLAB GUI}

I used MATLAB to control the Arduino and to extract and manipulate the raw data so that it can be easily analyzed. The Arduino performs several different measurements at once and the computer to which the Arduino is connected sends these measurements to a file, which can be used later for further analysis.

The MATLAB code I developed has a graphical user interface (GUI), as shown in Figure 7, which allows the users to label and change the parameters and their units. As soon as the GUI starts collecting data, the parameter information is written to a documentation file. In addition, the users can run the code in two modes. One mode continuously plots the nearly instantaneous values (there is some time delay between the Arduino getting a sensor value and the computer receiving the value from the Arduino) while the other mode collects data up for a user-specified duration and plots the average. When the GUI updates the plot, it displays the mean value of each of the measured parameters. When the Arduino has collected a data point from a particular input and has converted it to a meaningful value, the MATLAB code writes the value to a file with a time stamp.

\section{Methods used in the Cyclohexadiene Collaboration}

A velocity map imaging (VMI) spectrometer was used to detect the ions of the Coulomb exploded cyclohexadiene ring, as well as to record their time of flight. The VMI consists of two oppositely charged plates above and below the interaction region called the repeller and the extractor, respectively. To detect the positive ions, the repeller is positively charged while the extractor is negatively charged. The ions are accelerated through a hole in the 
extractor plate and past a lens system and a drift tube before they hit a micro-channel plate (MCP)[10]. Because the signal of a single ion is too weak to be detected, it is amplified by the MCP. The MCP has a number of small channels through which electrons can travel. When an ion hits the MCP, an electron is emitted and multiplied by repeatedly being accelerated and hitting the channel wall. The electron shower has ample charge to generate a fluorescence on the phosphorous screen behind the MCP. Though the phosphorous screen is showing electrons, it is recording where the ions have hit. A camera records images of the screen at the repetition rate of the LCLS.

Since this repetition rate is around $100 \mathrm{~Hz}$, a minute of the experiment will generate about 60,000 images. A single image is a relatively large file, so if one runs the experiment for many hours over several days, one amasses enormous amounts of data. The amount of data needs to be reduced in order to maximize data analysis efficiency.

The camera images are stored as very large .xtc files. To reduce the amount of collected data, a $\mathrm{C}$ program finds the $\mathrm{x}$ - and $\mathrm{y}$-positions of the ion hits and stores them as smaller .dat files. We wrote a MATLAB script that takes these .dat files, picks out ions and sorts these ion hits by a variety of parameters such as the delay between the UV and the LCLS pulses or the energy of the LCLS pulse that produced the ion hits. This is necessary because the delay and pulse energy as well as other parameters fluctuate at LCLS on a shot to shot basis. Binning is accomplished by splitting the difference between the highest and lowest recorded time delay between the UV and LCLS pulses into a certain number of segments of equal size. Each of these time delays segments represent a bin. Any single shot with a time delay that falls within a particular bin range is sorted into that bin. For example, if a shot has an associated time delay of 210 femtoseconds, the ions recorded in this shot are sorted into the 200-250 femtosecond bin. When the code is finished binning, the data is reduced to time delays and the number of ion hits now associated with these time delays. This 
sorting technique minimizes the amount of data taken during the LCLS run. In addition to fluctuating time delay, the LCLS pulse energy also fluctuates, so the data is further reduced by filtering out the ion hits that were produced with a pulse energy below a particular level.

\section{RESULTS}

\section{Pressure vs. HHG Yield}

As previously mentioned, the gas pressure in the chamber fluctuates and the HHG yield depends on the pressure. Since the Arduino-GUI system can read in and log sensor values with satisfactory speed, we can monitor the fluctuating pressure more closely. The HHG intensity is detected by a camera. The resulting measurement is time-stamped. Having

a time-stamp on the pressure measurements collected by the Arduino enables us to match HHG yield with the corresponding pressure with considerably less difficulty. Figure 8 shows a graph of the pressure versus HHG yield.

\section{Results in Ion Binning}

The MATLAB code we wrote was able to pick out the ions successfully. In particular, binning by time delay has helped reduce the amount of data. We are able to provide results from further analysis that have utilized our code. Figure 9 shows that the C-code was indeed able to pick out single ion hits. This shows that the first stage in the data reduction worked. Then, these ion hits from the single shots were binned according to the time delay between UV and X-ray pulse. An image of the binned shots is shown in Figure 10. 


\section{DISCUSSION AND CONCLUSIONS}

\section{A Better Data Acquisition System}

Replacing the old data acquisition infrastructure with an Arduino board has improved data collection significantly, as data acquisition has become more flexible, user-friendly, and cost effective. The old code, which plotted the pressures with respect to time was not very user-friendly; however with the GUI, it is much easier to stop and restart data acquisition without having to close the GUI window or restart MATLAB. An additional benefit is that the Arduino chip is a very reasonably priced microcontroller that has several useful applications for data acquisition, some of which have yet to be exploited.

\section{Controlling Components using the Arduino}

Given the versatile functionality of the Arduino, it will be able to control instruments in the lab or sound alarms based on the values being measured, in order to protect components like vacuum pumps from high pressure and other hazardous conditions. For this application,

the Arduino would not necessarily be connected to the computer. Therefore, the code should be written onto the Atmel chip as before, but the Arduino would not interact with a computer-controlled program. The code should be able to take the different undesirable scenarios into account and control different components of the experiment accordingly.

\section{Further Work in the Cyclohexadiene Collaboration}

The MATLAB code we wrote kept the more important pieces of information related to the time delays and the higher pulse energies, while eliminating enormous amounts of excess data, proving that this binning technique we utilized was every efficient. Due to promising preliminary results using the MATLAB code, it will be implemented to sort with different parameters and reduce the amount of unnecessary data. 


\section{ACKNOWLEDGMENTS}

I would like to thank my mentors Markus Gühr and Brian McFarland as well as Joe Farrell, Max Chalfin, and the rest of the Bucksbaum group for their guidance and support

during my summer. In addition, I would like to thank SLAC and the DOE for providing the funding that made this amazing opportunity possible. Finally, I would like to thank my fellow SULI interns for a wonderful summer.

\section{REFERENCES}

[1] P. B. Corkum, "Plasma perspective on strong field multiphoton ionization," Phys. Rev. Lett., vol. 71, pp. 1994-1997, 1993.

[2] K. J. Schafer, B. Yang, L. F. DiMauro, and K. C. Kulander, "Above threshold ionization beyond the high harmonic cutoff," Phys. Rev. Lett., vol. 70, pp. 1599-1602, 1993.

[3] J. L. Krause, K. J. Schafer, and K. C. Kulander, "High-order harmonic generation from atoms and ions in the high intensity regime." Phys. Rev. Lett., vol. 68, no. 24, pp. 3535 $-8,1992$.

[4] B. McFarland, J. Farrell, P. Bucksbaum, and M. Gühr, "High harmonic generation from multiple orbitals in $N_{2}, "$ Science, vol. 322, p. 1232, 2008.

[5] A. Eppink and D. Parker, "Velocity map imaging of ions and electrons using electrostatic lenses: Application in photoelectron and photofragment ion imaging of molecular oxygen," Rev. of Sci. Instrum., vol. 68, pp. 3477-3484, 1997.

[6] J. Feldhaus and B. Sonntag, "Free-electron lasers high-intensity x-ray sources," in Strong Field Laser Physics, ser. Springer Series in Optical Sciences, T. Brabec, Ed. Springer Berlin / Heidelberg, 2008, vol. 134, pp. 91-107. 
[7] doc8161.pdf. [Online]. Available: http://www.atmel.com/dyn/resources/prod_ documents/doc8161.pdf

[8] Arduino - ArduinoBoardDuemilanove. [Online]. Available: http://arduino.cc/en/ Main/ArduinoBoardDuemilanove

[9] "Arduino - Introduction," http://arduino.cc/en/Guide/Introduction.

[10] D. Rolles, Z. Pesic, M. Perri, R. Bilodeau, G. Ackerman, B. Rude, A. Kilcoyne, J. Bozek, and N. Berrah, "A velocity map imaging spectrometer for electron-ion and ion-ion coincidence experiments with synchrotron radiation," Nuclear Instruments and Methods in Physics Research Section B: Beam Interactions with Materials and Atoms, vol. 261, pp. $170-174,2007$.

[11] H. Frank, "File: FEL principle.png - Wikipedia, the free encyclopedia," http://en. wikipedia.org/wiki/File:FEL_principle.png. 


\section{FIGURES}

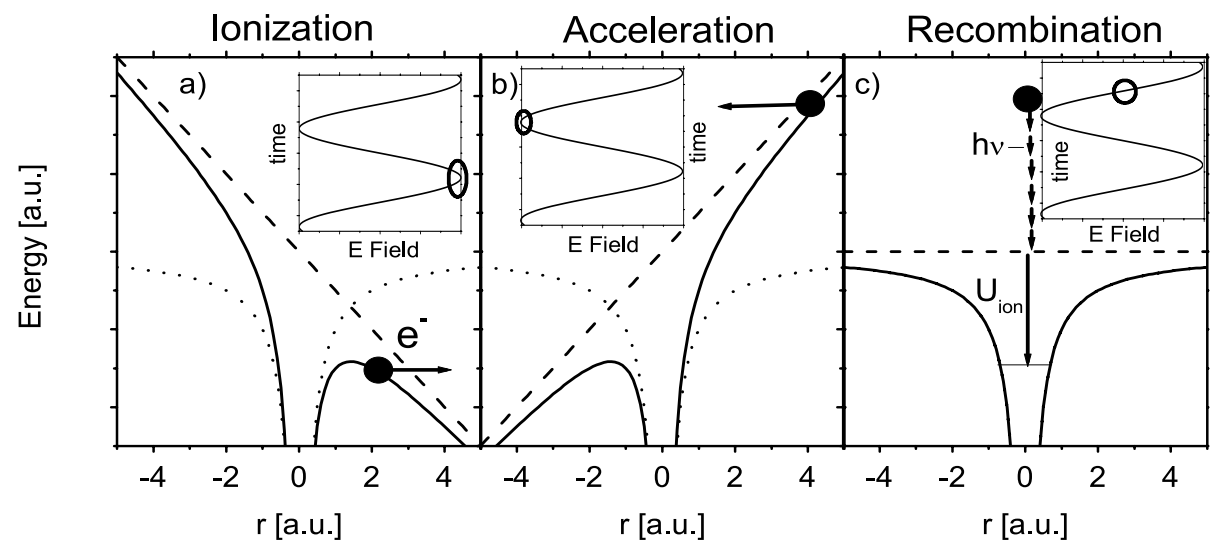

Figure 1: The dashed line represents the electric field potential, while the dotted line represents the Coulomb potential of an atom. The sum of the two potentials is represented by the solid line. The inset shows where the atom is with respect to the electric field phase. For more explanation see text.

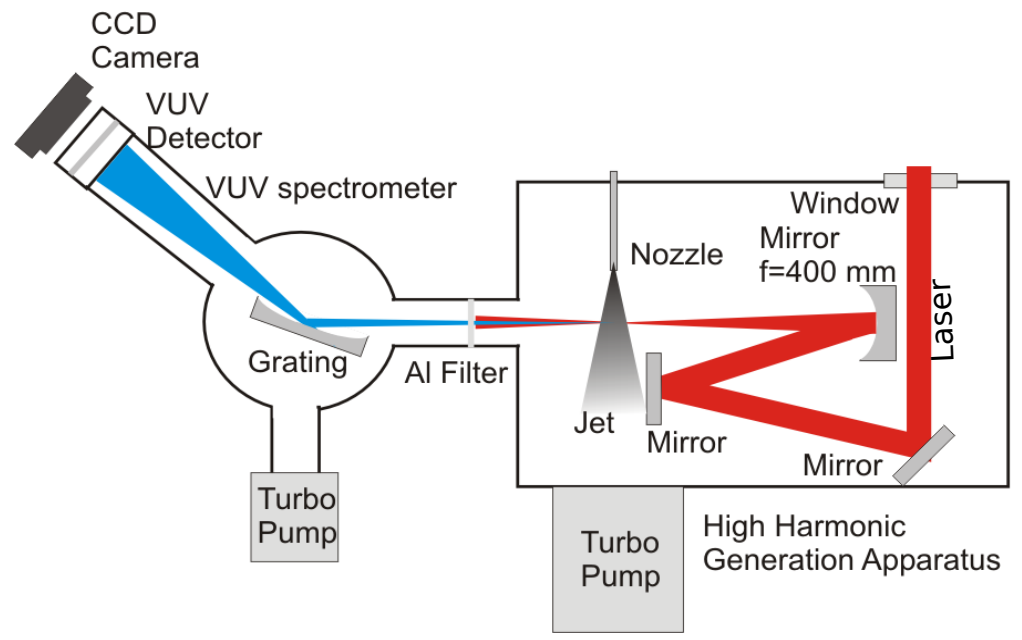

Figure 2: General set up for the experiment. The laser system is omitted and only the vacuum chambers containing the harmonic generation and the spectrometer are shown. The parameters that we can monitor with the Arduino are the laser power and the pressure of the gas in the jet before it enters the chamber. 


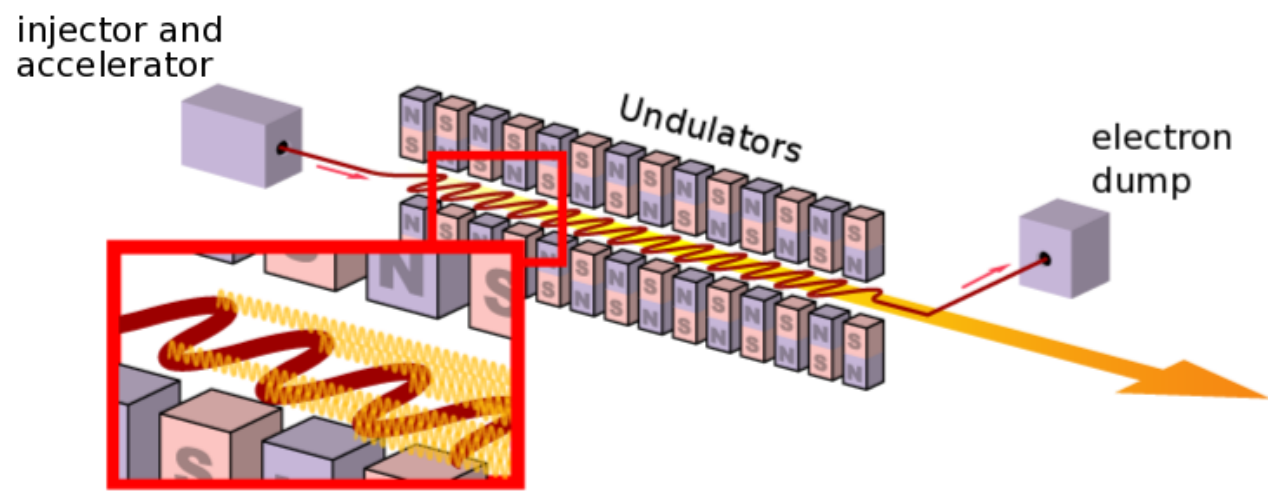

Figure 3: A layout [11] of the x-ray free electron laser at LCLS. Electrons are accelerated into the undulators where x-ray radiation is emitted whenever an electron changes direction. After they pass through the undulators, the electrons are sent to the electron dump while the x-rays continue to the experimental halls to be used. 


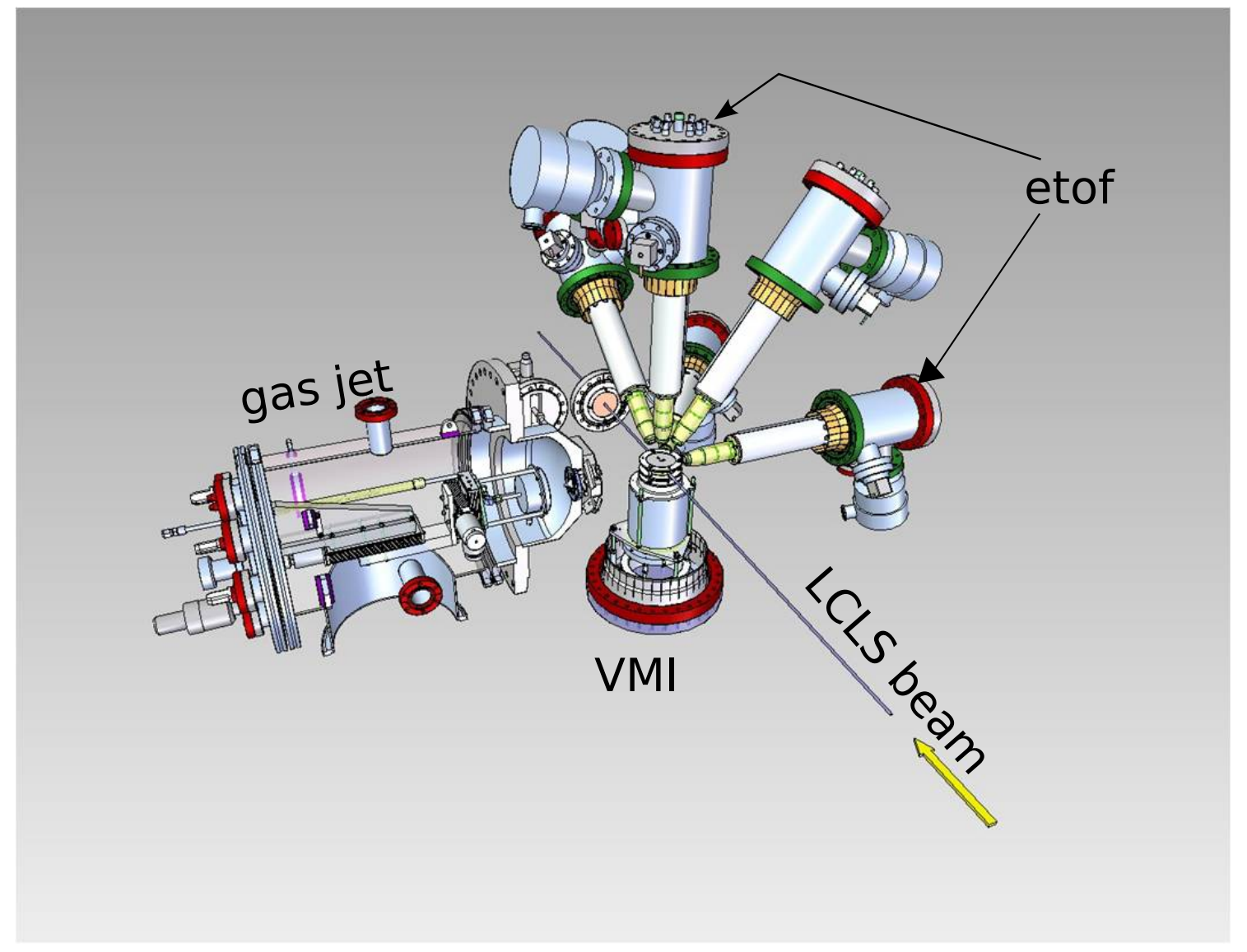

Figure 4: General setup of the AMO end station at LCLS. When the gas jet and the LCLS beam interact, the information about the ions are collected by the velocity map imaging (VMI) spectrometer. (Picture see John Bozek) 


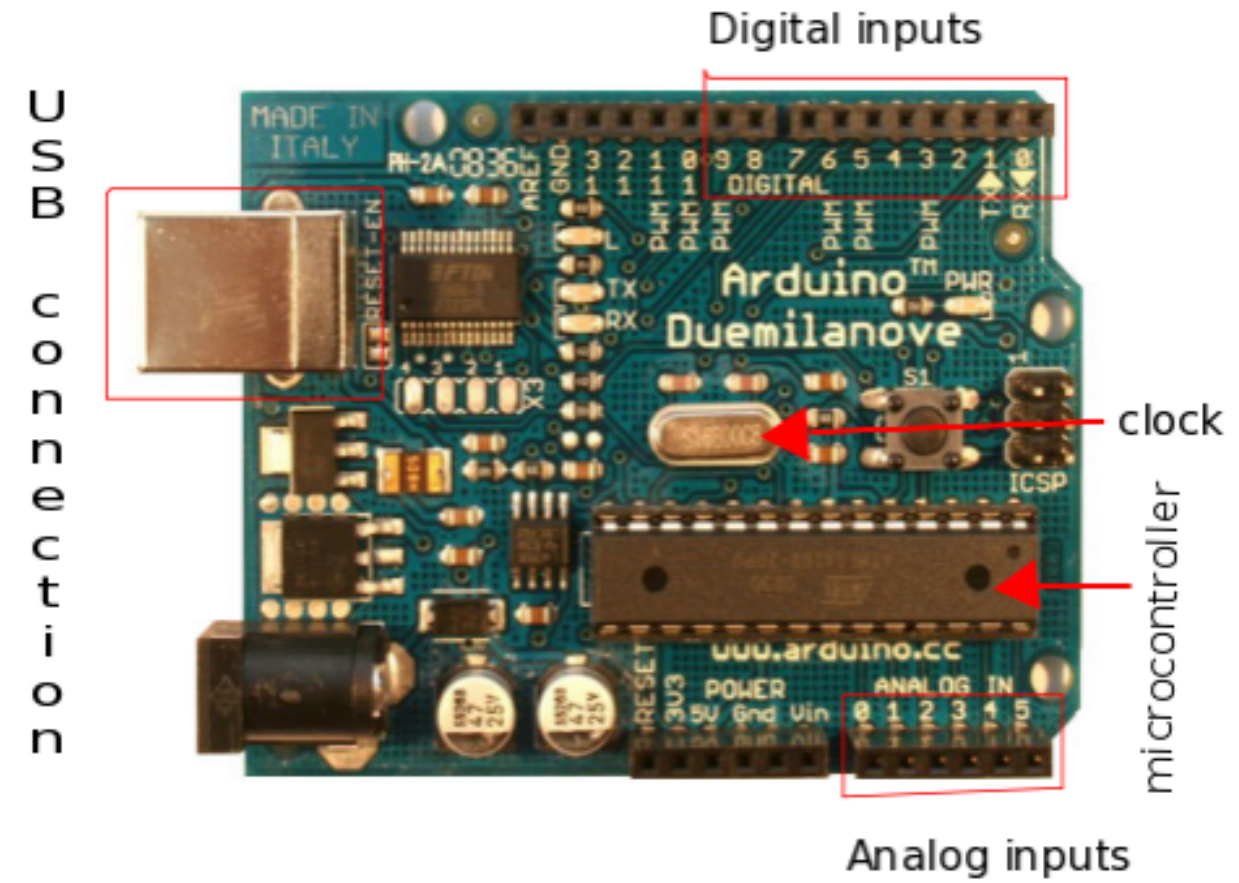

Figure 5: The Arduino Duemilanove has 6 analog inputs and 14 digital inputs.

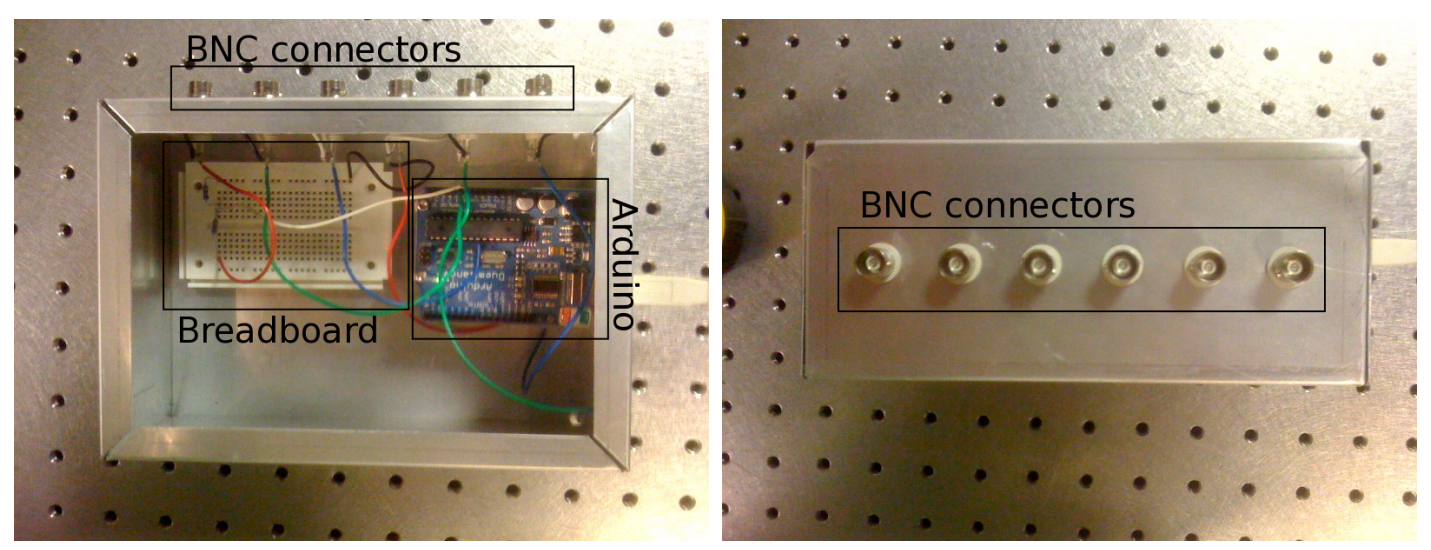

Figure 6: The photo on the left shows the Arduino and a little breadboard in the compartment. The breadboard is used to hold circuits to precondition the incoming voltage signal. The Arduino is on stands so that the bottom does not touch metal box. The photo on the right shows the box from the side to show the 6 BNC connectors. 


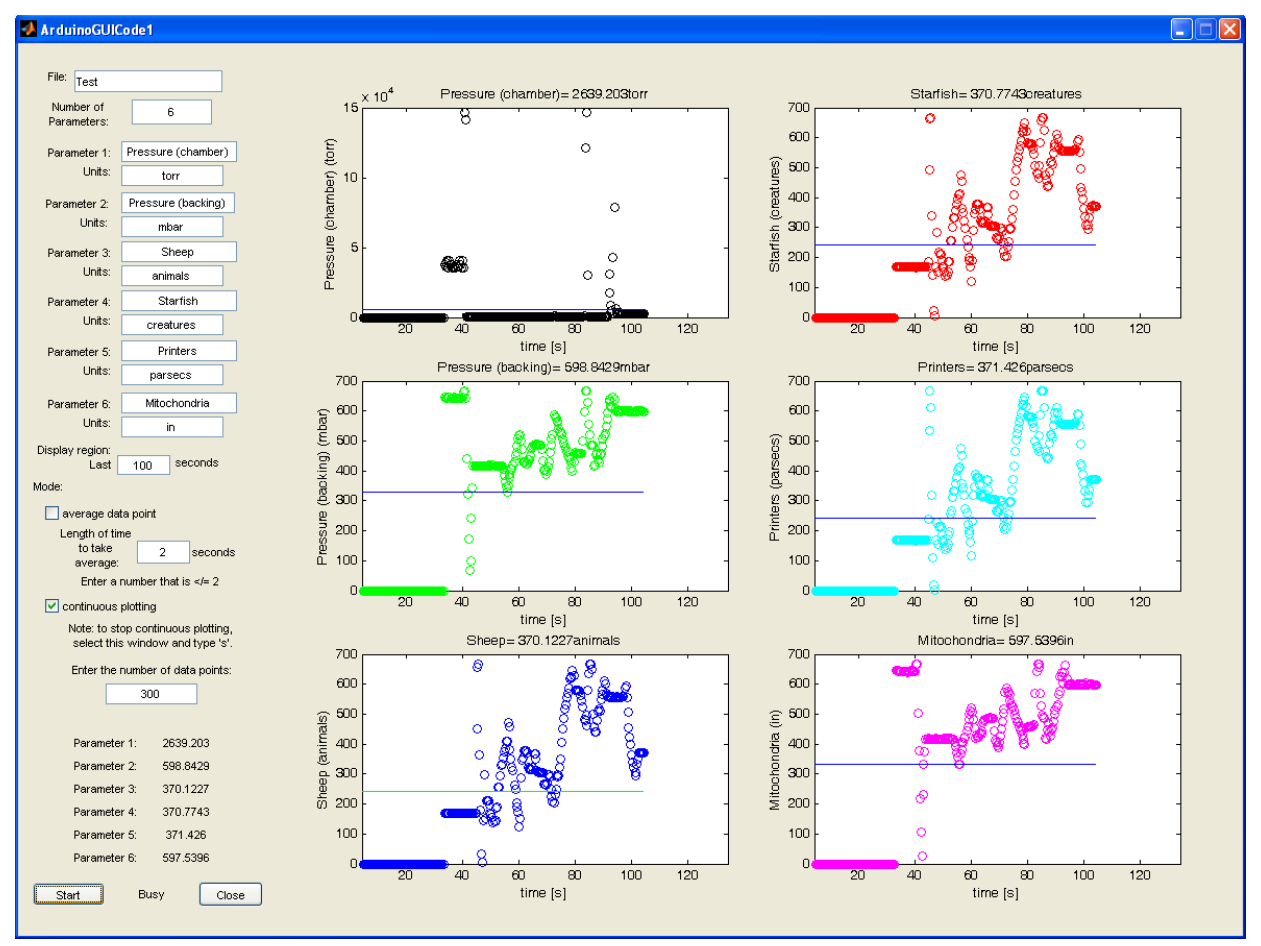

Figure 7: The GUI above is in continuous plotting mode and is monitoring six inputs.

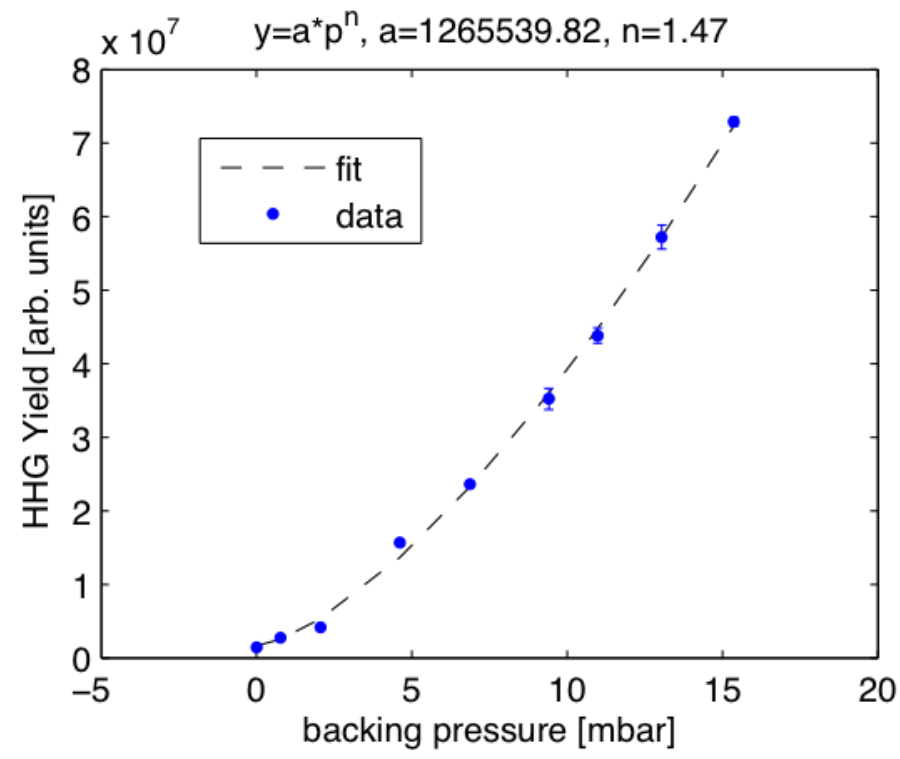

Figure 8: Pressure versus HHG Yield. In an ideal case of perfect phase matching, n would be 2 , meaning the signal would increase quadratically with pressure. 


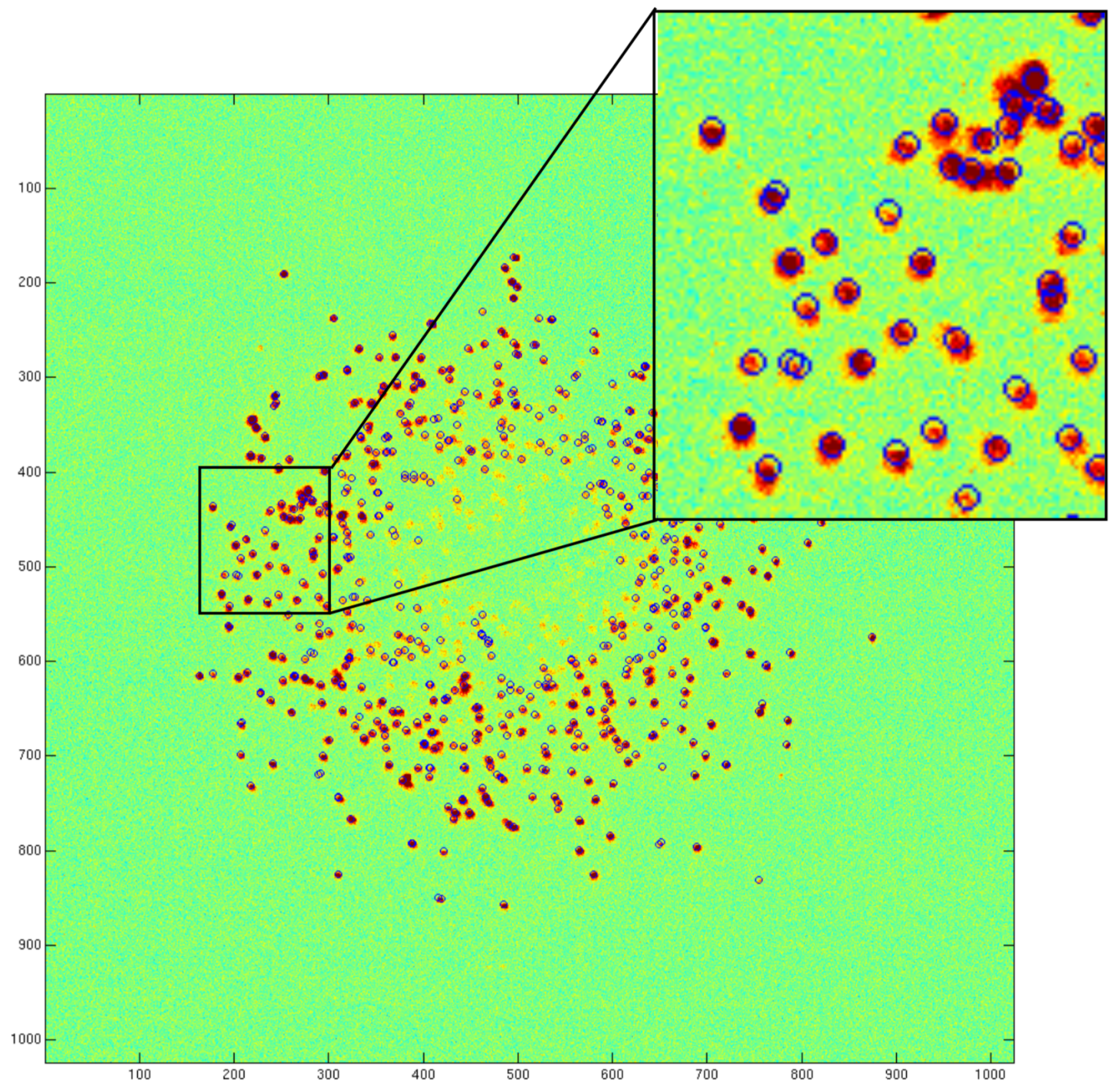

Figure 9: Image processed by $\mathrm{C}$ to pick out the ions hitting the VMI (blue circles). One can see that spots of signal intensity are treated as single ion hits. MATLAB is used to superimpose the image and the ions picks from the $\mathrm{C}$ code. 
Motorposition199.36 mm

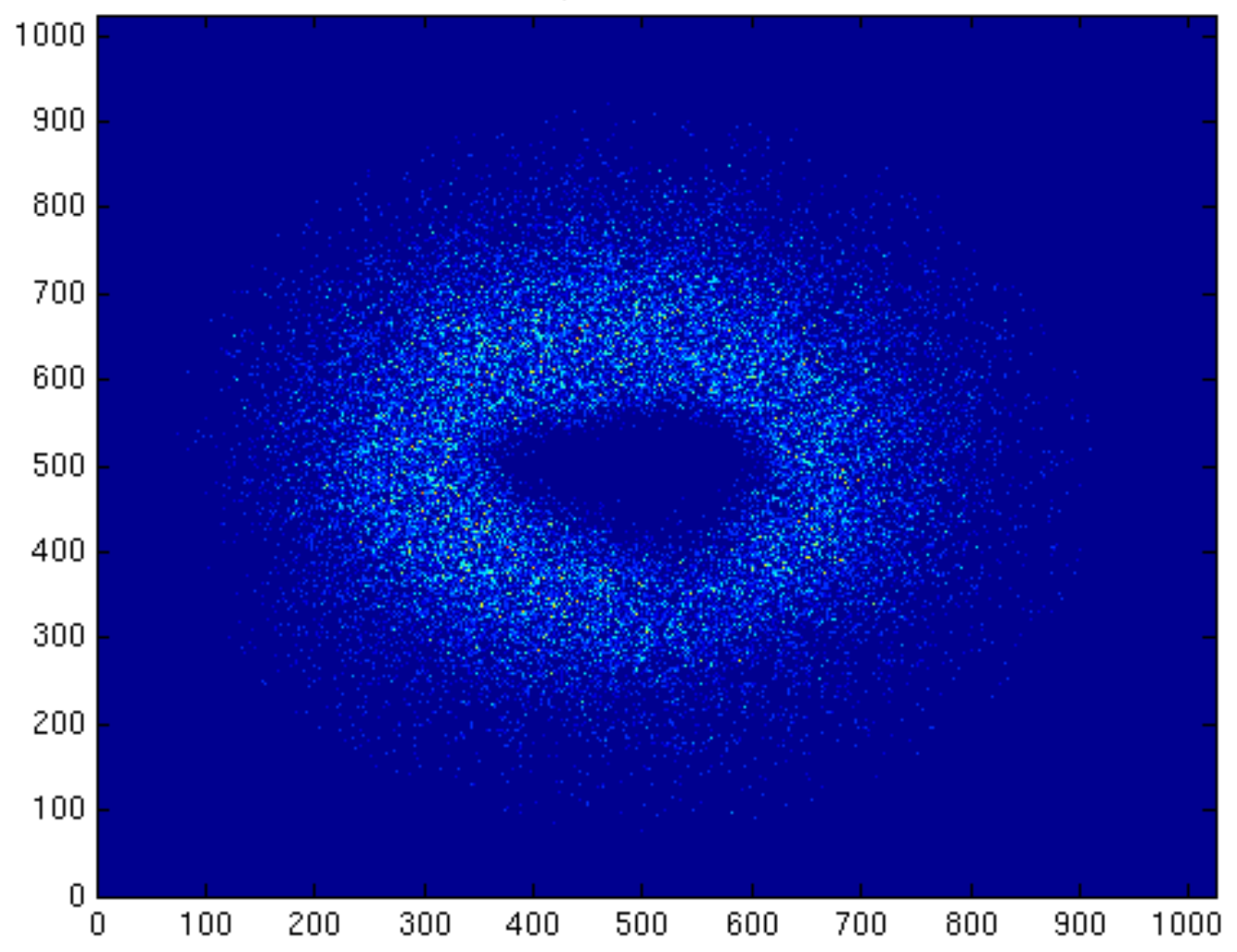

Figure 10: Ion hits of a particular time delay. The Motorposition defines the delay between the UV and the LCLS pulses. 